\title{
Solving the Power Purchase Cost Optimization Problem with Improved DE Algorithm
}

\author{
Jie Xiong \\ School of Electronics and Information \\ Yangtze University \\ Jingzhou, China
}

\author{
Caiyun Liu \\ School of Mathematics and Information \\ Yangtze University \\ Jingzhou, China
}

\begin{abstract}
Under the deregulation of generation market in China, all distributed generators will particular in electric power bidding. Therefore power purchase cost optimization (PPCO) problem has been getting more attention of power grid Company. However, under the competition principle, they can purchase power from several of power plants, therefor, there exist continuous and integral variables in purchase cost model, which is difficult to solve by classical linear optimization method. An improved differential evolution algorithm is proposed and employed to solve the PPCO problem, which targets on minimum purchase cost, considering the supply and demand balance, generation and transfer capability as constraints. It yields the global optimum solution of the PPCO problem. The numerical results show that the proposed algorithm can solve the PPCO problem and saves the costs of power purchase. It has a widely practical value of application.
\end{abstract}

Keywords-Power market; Power purchase cost optimization; Improved Differential Evolution; Adaptive parameter

\section{INTRODUCTION}

With the implementation of separating the power plants from grid company and generation bidding in power market in China, it is important for the grid company to optimize the purchasing schedule to reduce the cost of grid company[1-3].

According to the different settlement rules, the objective function of generation-side electricity market is divided to two classes. One is to minimum the cost based on the unified marginal settlement, while the other is minimum cost based on the practical generators bidding[4,5]. The second power purchase cost optimization(PPCO) problem is studied in this paper. The PPCO problem is studied for some years. DUAN established a purchase optimization model considering the generation capability and line loss, and solved this model by penalty function [1]. But he only discussed the PPCO problem on the protection and coordination principle. CHEN et al. established purchase optimization model on the market principle and solved it by Lagrange relaxation method [4]. TAN et al. discussed the multilevel purchase optimization model of long-term, mid-term and short-term electricity market and analyzed the relationship of them, but they did not give the solution method [6]. ZHANG et al. established a purchase optimization model with the constraint of supply and demand balance and constraint of generation capability. They solved this model by the PSO but they did not consider the transfer capability constraint and did not satisfy the constraint of supply and demand balance [2]. ZHANG et al. established a purchase optimization model with the constraints of supply and demand balance, generation capability and transfer capability. They solved it with PSO and obtain a satisfied solution [3].

Differential Evolution (DE) algorithm[7,8] is a heuristic global search method which has been employed in many fields such as chemical industry, machine design, information processing, biological information, geophysical inversion $[9,10,11]$. The DE algorithm has employed to solve the PPCO problem recently, but did not consider the constraints of transfer capability and supply and demand balance [12].

The purchase optimization model is established with the constraint of the supply and demand balance, generation capability, and transfer capability firstly. And an improved DE algorithm with the dynastic mutation factor and crossover factor is proposed secondly. Then the steps of algorithm for the PPCO problem based on the improved DE algorithm thirdly, and the correctness of the proposed algorithm is verified by numerical experiment.

\section{Power Purchase Model}

The goal of power purchase optimization is to minimum the cost of purchasing particular quantity of electricity considering some constraints.

The power purchase cost optimization problem can be modeled as follow:

$$
\begin{array}{ll}
\text { MinF, } & F=\sum_{i=1}^{n} C_{i} P_{i} \\
\text { S.T. } & \sum_{i=1}^{n}\left(1-\Delta P_{i}\right) P_{i}=P_{d} \\
& P_{i}=0 \text { or } P_{i \min } \leq P_{i} \leq P_{i \max } \\
& -L_{i \max } \leq L_{i} \leq L_{i \max }
\end{array}
$$


where $C_{i}, P_{i}, \Delta P_{i}$ is the electricity price, the purchase quantity of electricity and the line loss of $\mathrm{i}$-th power plant respectively; $P_{d}$ is the particular quantity of electricity should be purchased; $P_{i \min }$ and $P_{i \max }$ is the minimum and maximum generation capacity of $i$-th power plant respectively; $L_{i \max }$ is the transmission capacity of the line from $i$-th power plant to grid company; $F$ is the objective function of power purchase cost optimization.

\section{IMPROVED DE ALGORITHM}

\section{A. Basic DE algorithm}

DE algorithm first initialize is a parallel direct search method which utilizes $N P n$-dimensional parameter vectors $X^{0}=\left[x_{1}^{0}, x_{2}^{0}, \ldots, x_{N P}^{0}\right]$ as a population for each generation. The initial vector population is chosen randomly and should cover the entire parameter space. Then DE algorithm update population by the operation of mutation, crossover and selection.

(1) Mutation

DE generates new parameter vectors by the operation named mutation which add the weighted difference between two population vectors to a third vector.

$$
\boldsymbol{v}_{i}^{k+1}=\boldsymbol{x}_{r 1}^{k}+F\left(\boldsymbol{x}_{r 2}^{k}-\boldsymbol{x}_{r 3}^{k}\right)
$$

where $x_{i}^{k}=\left(x_{i 1}^{k}, x_{i 2}^{k}, \ldots, x_{i D}^{k}\right)$ is the $i$-th vector of $k$-th generation, $r_{1}, r_{2}, r_{3} \in\{1,2, \ldots, N P\}, r_{1} \neq r_{2} \neq r_{3} \neq i, F>0$ is the mutation factor.

(2) Crossover

In order to increase the diversity of the perturbed parameter vectors, crossover is introduced as follow.

$$
\boldsymbol{u}_{i j}^{k+1}=\left\{\begin{array}{l}
\boldsymbol{v}_{i j}^{k+1}, \operatorname{rand}(i j) \leq C R \text { or } j=\operatorname{rand}(j) ; \\
\boldsymbol{x}_{i j}^{k}, \operatorname{rand}(i j)>C R \text { or } j \neq \operatorname{rand}(j) ;
\end{array}\right.
$$

where $\operatorname{rand}(i j)$ is a uniform random number generator with outcome $\operatorname{rand}(i j) \in[0,1] ; C R$ is crossover factor $C R \in[0,1]$ which has to be determined by the user; $\operatorname{rand}(j)$ is a randomly chosen index $\operatorname{rand}(j) \in 1,2, \ldots, D$, which ensures that $\boldsymbol{u}_{i j}^{k+1}$ gets at least one parameter from $\boldsymbol{v}_{i j}^{k+1}$.

\section{(3) Selection}

To decide whether or not the $\boldsymbol{u}_{i j}^{k+1}$ should become a member of generation $k+1$, it is compared to the $\boldsymbol{x}_{i j}^{k}$ using the greedy criterion as follow.

$$
\boldsymbol{x}_{i}^{k+1}=\left\{\begin{array}{l}
\boldsymbol{u}_{i}^{k+1}, f\left(\boldsymbol{u}_{i}^{k+1}\right)<f\left(\boldsymbol{x}_{i}^{k}\right) ; \\
\boldsymbol{x}_{i}^{k}, \quad \text { Otherwise. }
\end{array}\right.
$$

If the algorithm convergence or the max iteration is achieved, algorithm finish, otherwise, begin the next iteration of mutation, crossover and selection.

\section{B. Improved DE Algorithm}

The mutation factor $F$ and crossover factor $C R$ is critical to the performance of basic $\mathrm{DE}$ algorithm. The large $F$ and $C R$ is beneficial to the global search ability, but it is harmful to the local search ability leading to the poor convergency speed, and vice versa. So we improve the DE algorithm in the following ways.

(1) Adaptive mutation factor

The following adaptive mutation factor is introduced in order to improve the global search ability at the early stage and the local search ability at the later stage of the algorithm.

$$
F=F_{\text {max }}-\frac{t\left(F_{\max }-F_{\min }\right)}{T_{\text {max }}}
$$

(2) Increasing crossover factor

DENG and RAN [13] propose the following strategy of increasing crossover factor to achieve the balance between global search ability at the early stage and local search ability in the later stage of the algorithm.

$$
C R=\left(C R_{\min }-C R_{\max }\right)\left[\left(\frac{t}{T_{\max }}\right)^{2}-2 \frac{t}{T_{\max }}+1\right]+C R_{\max }
$$

\section{(3) Random mutation}

In order to avoid the stagnation of $\mathrm{DE}$ algorithm at its last stage, a random mutation method is introduce. If an individual are stagnated in certain iterations, a random mutation is happen in this individual, that is random initializing a individual to replace the stagnated one. The random mutation method is described as follow:

if $F\left(x_{i}^{t}\right)=F\left(x_{i}^{t+1}\right)=F\left(x_{i}^{t+2}\right)=\cdots=F\left(x_{i}^{t+p}\right)$ and $F\left(x_{i}^{t}\right) \neq F^{*}$

then $x_{i}^{t+p+1}=x_{\text {min }}+\operatorname{rand}(0,1) *\left(x_{\max }-x_{\text {min }}\right)$

Where $F^{*}$ is fitness of global best individual, p is max stagnation iteration allowed, $\left(x_{\min }, x_{\max }\right)$ is the random search space.

We define the improved DE algorithm, which employ the adaptive mutation factor and increasing crossover factor strategy, named as DE1. And we define the improved DE algorithm, which employ the adaptive mutation factor, increasing crossover factor, and random mutation strategy, named as DE2. 


\section{SOLVING THE PPCO PROBLEM WITH IMPROVED DE ALGORITHM}

\section{A. Strategy for the constraints}

The generation and transfer capacity constraints are transformed to boundaries of parameter vector. The balance of supply and demand constraints is considered using the penalty function method and the PPCO problem is transformed from a optimization problem with constraints to a non-constraint optimization problem. The objective function is following:

$$
\operatorname{MinF}, F=\sum_{i=1}^{n} C_{i} P_{i}+\alpha\left[\sum_{i=1}^{l}\left(1-\Delta P_{i}\right) P-P_{\exp e c t}\right],
$$

where $P_{\text {expect }}$ is the particular power should be purchased; $\alpha$ is penalty factor; the variables be optimized $P_{i}$, is the power purchased from $i$-th power plant.

\section{B. Algorithm steps of improved DE for PPCO}

The algorithm steps of improved DE for PPCO problem are following:

Step 1: given the upper and lower boundaries of the variables be optimized $P_{i}$, the population $N P$, the upper and lower boundaries of mutation factor $F$ and crossover factor $C R$;

Step 2: initialize the population of parameter vector randomly according to the upper and lower boundaries of $P_{i}$;

Step 3: calculate the objective function value of $P_{i}$, according to formula (8);

Step 4: execute the mutation, crossover and selection according to formula (2),(3),(4) respectively, and obtain the next generation population $\left[\boldsymbol{x}_{1}^{k+1}, \boldsymbol{x}_{2}^{k+1}, \ldots, \boldsymbol{x}_{N P}^{k+1}\right]$;

Step 5: update the mutation factor and crossover factor according to the formula (5),(6);

Step 6: if the algorithm stagnate certain iterations, random mutation a individual according to the formula (7);
Step 7: calculate the objective function value of $P_{i}$, according to formula (8);

Step 8: if the convergency and the max iteration is not achieved, go to step 4, begin next iteration;

Step 9: output the solution, finish.

\section{NumERICAL RESUlt AND ANALYSIS}

\section{A. Benchmark Results}

We use following five benchmark functions to test our improved DE algorithm.

(1) Sphere

$$
f_{1}(x)=\sum_{i=1}^{n} x_{i}^{2}, \quad n=30,\left|x_{i}\right| \leq 100
$$

(2)Generalized Schwefel's

$$
f_{2}(x)=\sum_{i=1}^{n}\left(x_{i} \sin \left(\sqrt{\left|x_{i}\right|}\right)\right), n=30,\left|x_{i}\right| \leq 500
$$

(3)Rastrigr

$$
f_{3}(x)=\sum_{i=1}^{n}\left[x_{i}^{2}-10 \cos \left(2 \pi x_{i}\right)+10\right], n=30,\left|x_{i}\right| \leq 32
$$

(4)Griewank

$$
f_{4}(x)=\frac{1}{4000} \sum_{i=1}^{n} x_{i}^{2}-\prod_{i=1}^{n} \cos \left(\frac{x_{i}}{\sqrt{i}}\right)=1, n=30,\left|x_{i}\right| \leq 600
$$

(5)Ackly

$f_{5}(x)=-20 \exp \left[-0.2 \sqrt{\frac{1}{n} \sum_{i=1}^{n} x_{i}^{2}}\right]-\exp \left(\frac{1}{n} \sum_{i=1}^{n} \cos \left(2 \pi x_{i}\right)\right)+20+e$

, $n=30,\left|x_{i}\right| \leq 5.12$

The parameters of standard DE algorithm are set as follows: $C R=0.9, F=0.5$. The Parameters of improved DE algorithm are set as follows: $C R_{\min }=0.1, C R_{\max }=0.9$, $F_{\text {min }}=0.3, F_{\text {max }}=1.2$. Both algorithms' number of individuals are 60, max iteration are 2000. The test results are listed in the table 1 .

TABLE 1. Benchmark on DE and improved DE algorithm

\begin{tabular}{|c|c|c|c|c|c|c|}
\hline & & $f_{1}$ & $f_{2}$ & $f_{3}$ & $f_{4}$ & $f_{5}$ \\
\hline \multirow{5}{*}{ DE } & Average & $6.47 \mathrm{e}-31$ & $-9.74 \mathrm{e}+3$ & 102.02 & $7.40 \mathrm{e}-4$ & $5.51 \mathrm{e}-15$ \\
\cline { 2 - 7 } & Stand. dev. & $1.33 \mathrm{e}-39$ & 900.94 & 35.19 & 0.0022 & $1.63 \mathrm{e}-15$ \\
\cline { 2 - 7 } & Best & $1.51 \mathrm{e}-32$ & $-1.12 \mathrm{e}+4$ & 10.94 & 0 & $4.45 \mathrm{e}-15$ \\
\cline { 2 - 7 } & Worst & $7.34 \mathrm{e}-30$ & $-7.43 \mathrm{e}+3$ & 183.66 & 0.0074 & $7.99 \mathrm{e}-15$ \\
\cline { 2 - 7 } & Runtime & 85.92 & 131.89 & 107.17 & 118.06 & 97.97 \\
\hline \multirow{4}{*}{$\begin{array}{c}\text { Improved } \\
\text { DE(DE1) }\end{array}$} & Average & $4.98 \mathrm{e}-5$ & $-1.26 \mathrm{e}+4$ & $3.32 \mathrm{e}-5$ & $1.77 \mathrm{e} 04$ & 0.0015 \\
\cline { 2 - 7 } & Stand. dev. & $9.42 \mathrm{e}-5$ & $2.30 \mathrm{e}+4$ & $5.59 \mathrm{e}-5$ & $2.29 \mathrm{e}-4$ & 0.0014 \\
\cline { 2 - 7 } & Best & $7.66 \mathrm{e}-8$ & $-1.26 \mathrm{e}+4$ & $8.63 \mathrm{e}-9$ & $8.39 \mathrm{e}-8$ & $8.85 \mathrm{e}-5$ \\
\cline { 2 - 7 } & Worst & $2.43 \mathrm{e}-4$ & $-1.26 \mathrm{e}+4$ & $3.08 \mathrm{e}-4$ & $8.67 \mathrm{e}-4$ & 0.0055 \\
\hline \multirow{2}{*}{$\begin{array}{l}\text { Improved } \\
\text { DE(DE2) }\end{array}$} & Runtime & 262.24 & 260.94 & 278.27 & 279.52 & 284.47 \\
\cline { 2 - 7 } & Average & $4.13 \mathrm{e}-5$ & $-1.26 \mathrm{e}+4$ & $1.67 \mathrm{e}-5$ & $8.23 \mathrm{e}-05$ & 0.0012 \\
\hline
\end{tabular}




\begin{tabular}{|c|c|c|c|c|c|c|}
\hline & Best & $9.61 \mathrm{e}-12$ & $-1.26 \mathrm{e}+4$ & $7.59 \mathrm{e}-10$ & $2.02 \mathrm{e}-8$ & $2.14 \mathrm{e}-5$ \\
\cline { 2 - 7 } & Worst & $4.72 \mathrm{e}-4$ & $-1.26 \mathrm{e}+4$ & $3.01 \mathrm{e}-4$ & $4.89 \mathrm{e}-4$ & 0.0050 \\
\cline { 2 - 7 } & Runtime & 290.94 & 274.67 & 279.70 & 323.17 & 267.74 \\
\hline
\end{tabular}

The results listed in table 1 indicate that the both DE1 and DE2 can improve the accuracy of fitness of benchmark functions, while DE2 is better than DE1 at small degree.

\section{B. PPCO Results}

The problem of grid company purchases 200GWh power from 5 power plant is considered. The model of power supply is illustrated as Figure 1.

There is a backup line between plant 2 and 3, which is opened normally. When the line between plant 2 and grid company or between plant 3 to grid is broken, the backup line is closed and the plant 2 and plant 3 share one line to transfer the power. The line loss of the backup line between plant 2 and plant 3 is 0.002 .

The emulation program is written using Matlab $\mathrm{m}$ language. The parameters are chosen as following: $\mathrm{D}=5, N P=40, F_{\text {min }}=0.3, F_{\max }=1.2, C R_{\min }=0.1, C R_{\max }=0.9, \quad \max$ iteration is 5000. The price, line loss, generation and transfer capability are listed in table 2.

Under the normal condition, that means the transfer capability is larger than the generation capability. The results of purchase optimization under the protection and coordination principle are listed in the table 3, compared with the results of literature [2][3][4]. The results show that the cost of our algorithm is same as that of literature [3], but larger than those of the literature [2][3] slightly, because the results of ours and literature [3] is obtained under the constraint of supply and demand balance restrictively whereas those of literature [2][4] is obtained with some deviation of it.

The results of purchase optimization under the normal condition and marketing principle are listed in the table 4, compared with the results of literature [2][3][4]. The results show that the cost of our algorithm is same as those of literature [3][4] which are satisfied with the constraint of supply and demand balance restrictively, while the result of literature [2] is not satisfied that constraint restrictively.

When the line between plant 3 and grid company is broken, the backup line between plant 2 and 3 is close and the two plants share one transfer line between plant 2 and grid company. The results of purchase optimization under such the abnormal condition are listed in the table 5. The optimization results are same as those of literature [3]. Under the protection and coordination principle is 27.6789 million Yuan( $¥)$, and under the marketing principle is 27.3541 million Yuan( $(Y)$.

The numerical results show that the proposed improved DE algorithm has the advantage of fine global optimize ability. It can be employed to solve the PPCO problem and reduce the purchase cost obviously.

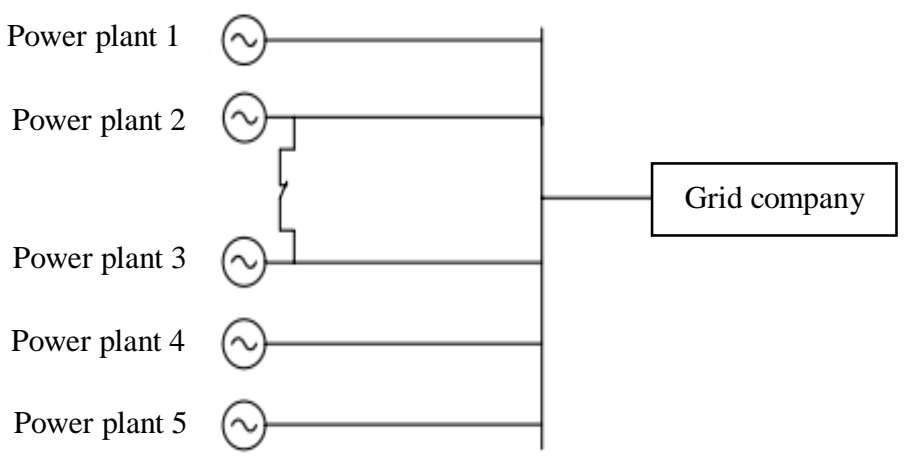

Fig. 1. power supply model of 5 power plants

TABLE 2. Parameters of power supply model of 5 power plants

\begin{tabular}{|c|l|l|l|l|l|}
\hline & $C_{i}(¥ / \mathrm{kWh})$ & $\Delta P_{i}$ & $P_{\text {inin }} / G W h$ & $P_{i \max } / G W h$ & $L_{i \max } / G W h$ \\
\hline plant 1 & 0.10 & 0.0882 & 43.2 & 86.4 & 100 \\
\hline plant 2 & 0.12 & 0.0722 & 21.6 & 64.8 & 90 \\
\hline plant 3 & 0.15 & 0.0451 & 21.6 & 43.2 & 60 \\
\hline plant 4 & 0.18 & 0.0422 & 14.4 & 43.2 & 60 \\
\hline plant 5 & 0.20 & 0.0554 & 14.4 & 28.8 & 40 \\
\hline
\end{tabular}

TABLE 3. The results of power purchase optimization under the protection and coordination principle

\begin{tabular}{|l|l|l|l|l|}
\hline & literature[2] & literature[3] & literature[4] & our results \\
\hline plant 1 & 86.4000 & 86.4000 & 86.3979 & 86.4000 \\
\hline plant 2 & 64.8000 & 64.8000 & 64.6384 & 64.8000 \\
\hline plant 3 & 35.2963 & 35.6356 & 35.2467 & 35.6356 \\
\hline plant 4 & 14.4000 & 14.4000 & 14.5145 & 14.4000 \\
\hline plant 5 & 14.4000 & 14.4000 & 14.4549 & 14.4000 \\
\hline cost/million Y & 27.1825 & 27.2333 & 27.19498 & 27.2333 \\
\hline supply/demand balance & 199.6760 & 200.0000 & 199.6391 & 200.0000 \\
\hline
\end{tabular}


TABLE 4 . The results of power purchase optimization under the marketing principle

\begin{tabular}{|c|l|c|l|l|}
\hline & literature[2] & literature [3] & literature[4] & our results \\
\hline plant 1 & 86.4000 & 86.4000 & 86.4000 & 86.4000 \\
\hline plant 2 & 64.8000 & 64.8000 & 64.8000 & 64.8000 \\
\hline plant 3 & 43.2000 & 43.2000 & 43.2000 & 43.2000 \\
\hline plant 4 & 20.7218 & 21.0601 & 21.0601 & 21.0601 \\
\hline plant 5 & 0.0000 & 0.0000 & 0.0000 & 0.0000 \\
\hline cost/million Y & 26.6269 & 26.6868 & 26.6868 & 26.6868 \\
\hline $\begin{array}{c}\text { Supply/demand } \\
\text { balance }\end{array}$ & 199.6760 & 200.0000 & 200.0000 & 200.0000 \\
\hline
\end{tabular}

TABLE 5. The results of power purchase optimization under the abnormal condition

\begin{tabular}{|c|c|c|c|c|}
\hline & \multicolumn{2}{|l|}{ literature [3] } & \multicolumn{2}{|l|}{ our results } \\
\hline & $\begin{array}{l}\text { protection } \\
\text { and coordination } \\
\text { principle }\end{array}$ & principle & $\begin{array}{l}\text { protection and } \\
\text { coordination } \\
\text { principle }\end{array}$ & $\mathrm{d} \underset{\text { marketing }}{\text { principle }}$ \\
\hline plant 1 & 86.4000 & 86.4000 & 86.4000 & 86.4000 \\
\hline plant 2 & 64.8000 & 64.8000 & 64.8000 & 64.8000 \\
\hline plant 3 & 25.2000 & 25.2000 & 25.2000 & 25.2000 \\
\hline plant 4 & 25.5659 & 39.7675 & 25.5659 & 39.7675 \\
\hline plant 5 & 14.4000 & 0.0000 & 14.4000 & 0.0000 \\
\hline cost/million $Y$ & 27.6779 & 27.3541 & 27.6779 & 27.3541 \\
\hline Supply/demand balance & 200.0000 & 200.0000 & 200.0000 & 200.0000 \\
\hline
\end{tabular}

\section{CONCLUSION}

An improved DE algorithm, which introduce new update method of mutation and crossover factor to improve the balance between the fine global search ability at the early stage and the fine local search ability at the later stage, is employed to solve the power purchase cost optimization (PPCO) problem with generation and transfer capability constraints. The results of five plants power supply model show that the proposed algorithm can find the global optimum of this PPCO problem and reduce the purchase cost obviously. It has a widely practical value of application.

\section{ACKNOWLEDGMENT}

This work is support by the National Science Foundation of China (No. 61273179, No. 61673006), and Science and Technology Research Project of Education Department of Hubei Province of China (No. D20131206, No. B2016034, No. 20141304).

\section{REFERENCES}

[1] S.Q. Duan, "Method for the power purchase cost optimization(in Chinse)", Proceeding of International Conference of Shanghai Grid[C], Shanghai, China, 2000, pp.619-621.

[2] T. Zhang, J.D. Cai, "Application of Improved Particle Swarm Optimization in Power Purchase Model Optimization Problem(in Chinese)". High voltage engineering, 2006, Vol. 32, No. 11, pp.131-134.

[3] L. Zhang, L. Lv, "Solving bidding model by using particle swarm optimization for power market(in Chinese)". Power system protection and control, 2009, Vol.37, No.22, pp.49-53.
[4] S.B. Chen, Z.H. Ding, "Solving bidding model by using Lagrange relaxation method for power market(in Chinese)". Proceeding of the EPSA, 2002, Vol.14, pp.76-78.

[5] E.K. Yu, J.Y. Zhou, X.S. Zhang, "Bidding Model and Bidding Principle for Power Markets(in Chinese)", Automation of Electric Power Systems, 2001, Vol. 25, No. 1, pp.24-27.

[6] Z.F. Tan, L.H. Zhang, X.J. Li, et al., "On Multilevel Purchasingelectricity Model for Electricity Grid Company in Region Electricity Market(in Chinese)", Journal of Electric Power, 2004, Vol. 19, No.4, pp.273-275.

[7] S. Storn, and K. Price, "Differential Evolution - A Simple and Efficient Heuristic for Global Optimization over Continuous Spaces", Journal of Global Optimization, 1997, Vol. 11, No. 4, pp.341-359.

[8] S. Das, S.S. Mullick, P.N. Suganthan, "Recent advances in differential evolution - An updated survey", Swarm and Evolutionary Computation, April 2016, Vol. 27, pp.1-30.

[9] A. Singh, S. Kumar, "Differential evolution: an overview", Advances in Intelligent Systems and Computing, 2016, Vol. 436, pp.209-217.

[10] F. Neri, and V. Tirronen, "Recent advances in differential evolution: a survey and experimental analysis", Artificial Intelligent Review, 2010, Vol. 33, pp.61-106.

[11] J. Xiong, C. Zou, "Induction logging inversion algorithm based on differential evolution(in Chinese)", Journal of Computer Applications, 2014, Vol. 34, No. 3, pp. 911-914.

[12] T. ZHANG, and H.J. RAN, "Application of differential evolution algorithm in purchase cost optimization", 2011, MACE 2011 Proceedings, Inner Mongolia, China.

[13] Z.X. Deng, X.J. Liu. "Study on strategy of increasing cross rate in differential evolution algorithm(in Chinese)", Computer Engineering and Application, 2008, Vol. 44, No.27, pp.33-36. 


\section{AUTHORS PROFILE}

Jie Xiong received his Ph.D. Degree in Geophysics and Information Technology from China University of Geosciences in 2012. He currently is an Associate Professor at School of Electronics and Information, Yangtze University, China. His research interests include power system automation, geophysical inversion theory, cloud computing, and scientific visualization.

Caiyun Liu (corresponding author) received her Ph.D. Degree in Geophysics and Information Technology from China University of Geosciences in 2014. She currently is an Associate Professor at School of Mathematics and Information, Yangtze University, China. Her research interests include applied geophysics, wavelet analysis, and optimization theory.

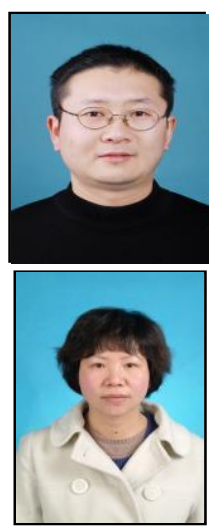

(c) 2017 by the author(s); licensee Empirical Research Press Ltd. United Kingdom. This is an open access article distributed under the terms and conditions of the Creative Commons by Attribution (CC-BY) license. (http://creativecommons.org/licenses/by/4.0/). 